\title{
USING OPENLY SOURCED 3D GEOGRAPHIC INFORMATION SYSTEMS (GIS) IN DETERMINING THE PHOTOVOLTAIC POTENTIAL OF QUEZON CITY HALL IN TERMS OF RECEIVED DIRECT SOLAR RADIATION
}

\author{
M. L. R. Lagahit ${ }^{1,2, *}$, A. C. Blanco ${ }^{1}$ \\ ${ }^{1}$ Dept. of Geodetic Engineering, University of the Philippines Diliman, Quezon City, Philippines - acblanco@ up.edu.ph \\ ${ }^{2}$ Dept. of Geomatics, National Cheng Kung University, Tainan, Taiwan - miguellagahit@ prs.geomatics.ncku.edu.tw
}

\section{Commission IV}

KEY WORDS: 3D GIS, Photovoltaics, Solar Energy, Renewable Energy, LiDAR

\begin{abstract}
:
For the past few years the United Nations have been standing strong in advocating the 17 sustainable development goals. One of those goals focuses on affordable and clean energy, which includes renewable energy. This study focuses on the application of geomatics on renewable energy, more specifically solar energy. The research aims to determine the photovoltaic potential of Quezon City Hall in the Philippines by calculating the amount of direct solar radiation it receives through a process that uses only openly sourced 3D GIS (Geographic Information Systems). The methodology mainly consists of (1) generating a building model composed of points that contains heights derived from a LiDAR (Light Detection and Ranging) based NDSM (Normalized Digital Surface Model) through the combined use of Python and QGIS, (2) determining the intersections between the building model and the sun's light rays, incorporating shadow factors and integrating solar irradiation values using Python, and (3) visualizing and gridding for analysis through the combined use of Blender, QGIS and a Spreadsheet software. Results have shown that Quezon City Hall has good photovoltaic potential since: solar irradiation values in 2017 has shown that the city hall receives $800 \mathrm{~W} / \mathrm{m}^{2}$ to $1000 \mathrm{~W} / \mathrm{m}^{2}$ on average from 08:00 to 16:00, and all faces of the building receive solar radiation. This means that all sides of the building can be proper candidates for solar panel or for Building Integrated Photovoltaics (BIPV) installations.
\end{abstract}

\section{INTRODUCTION}

\subsection{Background}

On January 1st 2016, the 17 Sustainable Development Goals (SDGs) of the 2030 Agenda for Sustainable Development of the United Nations became official. (United Nations, 2016) Goal \#7, of the SDGs, spearheads the agenda's motives on affordable and clean energy, such as the use of renewable energy. As of 2017 , the Philippines' total energy supply source is about $40 \%$ renewable, and $10 \%$ of that is supplied by solar, wind and biofuels (International Renewable Energy Agency, 2017).

Integration of renewable energy, especially solar energy, in urban areas is becoming crucial for its development (Ronzino, et al., 2015). City buildings' facades and rooftops offer an attractive option for solar energy (Brito, et al., 2017). As such, it is important to give a more detailed look in solar energy technologies such as photovoltaics that can be used on existing buildings (Kanters, et al., 2014). To unlock the potential of photovoltaics, research using Geographic Information Systems (GIS) is needed (Ronzino, et al., 2015). By integrating GIS in the decision-making process, photovoltaic systems can be properly located (Beseničar, et al., 2008) and maximized by processing spatial information in an urban context. (Ronzino, et al., 2015) Solar radiation models that has undergone GIS process provides accurate estimates of solar radiation over any surface (Adeleke et al., 2016). Currently it is already accepted that 3D city models are needed to determine solar resources in an urban context (Martin, et al., 2015). Although 3D models are generally used, previous studies on solar radiation on buildings at an urban scale only accounts for rooftops, to make it more comprehensive facades are also needed to be included (Wieland, et al., 2015).
This research aims to fill that gap, using 3D GIS methodologies to include both roofs and facades in determining photovoltaic potential in a building using openly sourced software and data.

\subsection{Objectives}

The main objective of this research is to determine the photovoltaic potential of Quezon City Hall. To accomplish the main objective, a subset of the following objectives must first be attained: (1) to determine solar irradiation values and its trend for the year of 2017, (2) to determine which faces of a building (roofs and facades) receives solar radiation, and (3) to determine the area coverage of solar radiation in the faces of a building.

\subsection{Area of Interest}

Figure 1 shows: (1) Quezon City Hall's Main Building and its extensions (outlined in red), and (2) The Hall of Justice (outlined in blue).

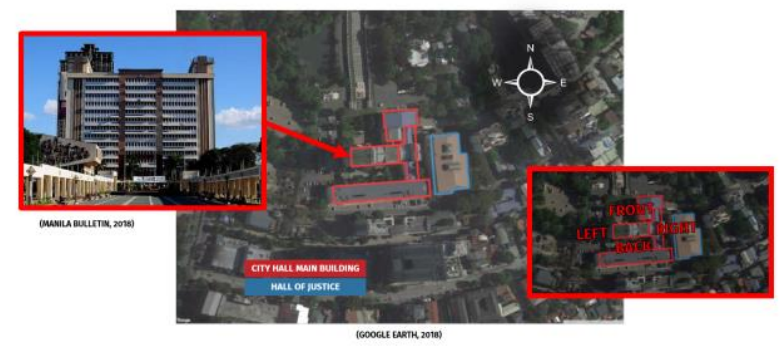

Figure 1. Google Earth Screenshot of Buildings in Area of Interest

\footnotetext{
* Corresponding author
} 
The area of interest is the main building of the Quezon City Hall. It is one of the highest and most expansive city halls in the country (Quezon City Government, 2018). The city hall has about 5,300 permanent employees and 5,800 contractual personnel and consultants (Quezon City Government, 2011). These buildings also contain offices that has daily transactions and services in availing and requesting for various permits, clearances and legal help. To incorporate shadow factor contributions, surrounding buildings, the main building's extensions and the building next to it, the Hall of Justice, are included in the processing.

\subsection{Scope and Limitations}

The research will generate a building model composed of points derived from LiDAR based NDSM. Photovoltaic potential will be measured through calculated solar positions and solar irradiance for every first day of the months of 2017. As such, the research is limited to the following: (1) The quality of the building models is highly dependent on the quality/resolution of the LiDAR based Digital Surface Model (DSM) and Digital Terrain Model (DTM). (3) Shadowing factors that are taken into consideration are only shadows that are produced by the buildings, this excludes vegetation, clouds, and etc. (4) Only direct solar radiation is taken into account, other types like diffused and reflected are omitted. (5) Light rays coming from the sun are treated as parallel since the light source is very far away (Zeng, 2018).

\section{REVIEW OF LITERATURE}

\subsection{Solar Energy}

2.1.1 Solar Energy and Photovoltaics: Solar Energy is energy harvested from the sun. Photovoltaics on the other hand is a way of harnessing solar energy, it is the process of using solar cells to convert light directly into electricity (McMordie, 2012). Photovoltaic potential can be determined through solar irradiance, the power density of solar radiation incident on a surface (Chwieduk, 2014).

2.1.2 Solar Radiation Components: Solar radiation has three components: direct, diffuse and reflected. Reflected radiation contributes the least of the three (Adeleke, et al., 2016) and is only assessable by very cost intensive calculations, thus it is often omitted in models. Diffuse radiation results from interactions with the cloud cover and vapor content of the lower atmosphere. However, since cloud cover data, vapor content and aerosols are difficult to assess it is also often omitted. Direct radiation is simply a direct contact with solar radiation (Jaugsch, et al., 2016). In this research only direct radiation will be observed.

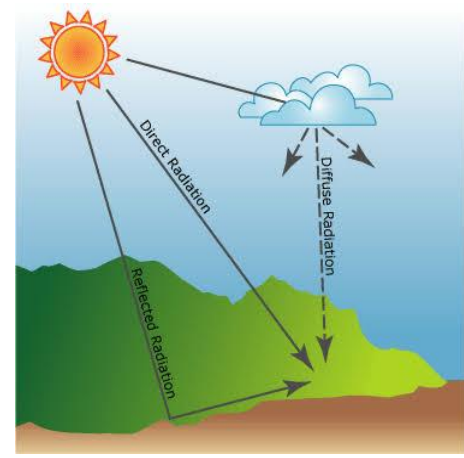

Figure 2. Solar Radiation Components $\odot$ ArcGIS Pro
2.1.3 Solar Radiation Factors: Solar radiation has the following factors: (1) the sun's location, (2) local topography, and (3) atmospheric attenuation (Miglani, et al., 2015). The sun's location is dependent on terrestrial geometry, which is the rotation and revolution of our planet around the sun. The sun's location determines the available solar radiation based on solar position above the horizon. For local topography, the shadowing effects of the surrounding terrain can modify the radiation distribution to the Earth's surface (Agugiaro, et al., 2011). Finally, atmospheric attenuation, which is caused by gas, solid and liquid particles (ex. clouds) in the atmosphere (Agugiaro, et al., 2011). This research takes into account the first two factors and takes the last factor up to a certain degree as provided by PySolar.

2.1.4 Clear and Real Sky: Clear sky radiation is radiation received by the surface after passing through the atmosphere without the presence of any clouds. Real sky radiation on the other hand, takes into account the cloud cover (Miglani, et al., 2015). This research estimates solar radiation under the terms of a clear sky radiation model.

\subsection{D GIS and Solar Energy}

2.2.1 3D Modelling: Due to the availability of LiDAR based data sets, more accurate three-dimensional city models can be produced. Having a better representation of building geometry is necessary in identifying where a solar panel could be installed (Martin, et al., 2015). since solar panels could both be installed on the roofs and the walls of a building, (Wegertseder, et al., 2016) since walls are larger in area than roofs and may also contribute to energy production (Jaugsch, et al., 2016). Indicating the Level of Detail of a generated building model is also important to determine the accuracy of solar radiation estimates, since complex geometries like the shape of roofs and wall extrusions are taken into account (Agugiaro, et al., 2011).

In the research of Jaugsch, et al., (2016) and the research of Wegertsder, et al., (2016) they used a model from extruded building footprints, that derived its height from a LiDAR based DSM. In another research done by Rafiee, et al., they used a more detailed building model considering the shape of the roof and the extrusions in the walls. In the case of the research of Martin, et al., (2015) the building model that they have used is the DSM itself. In this research hyper points based on a NDSM bounded by a building footprint will serve as the building model, which preserves the building roofs shape as well as the buildings irregular shape.

2.2.2 Observer Points and Hyper Points: Hyper points are points having the same $\mathrm{x}$ and $\mathrm{y}$ coordinate but different and varying $\mathrm{z}$ or elevation values (Jaugsch, et al., 2016). In the case of this research dense hyper points are used to model the buildings of interest. Observer points are points cover the surface of the model. It enables to make a quick estimate of solar radiation over an extent of a surface where it is located (Jaugsch, et al., 2016). For this research hyper points are on the outside or surface of the model will act as observer points.

In the research of Jaugsch, et al., they erected hyper points along the extruded building footprint boundaries, these acted as observer points which contained solar irradiance values of certain parts of the surface of the extruded building footprint. In this research the hyper points will serve as the building model and the observer points, which are hyperpoints on the surface, will serve as solar radiation model of Quezon City Hall at given date and time. 
2.2.3 Shadow Factors: Shadowing effects play an important role on estimating solar energy potential (Rafiee, et al., 2014). It is crucial to consider shadowing effects due to topography or shadows cast by nearby buildings, (Agugiaro, et al., 2011) after all, in an urban environment, shadowing effects causes the largest reduction of solar radiation. Modelling of shadowing effects is cost intensive in computing in an urban setting (Jaugsch, et al., 2016) and must be taken into consideration.

\subsection{PySolar}

PySolar is a python module that is aimed for photovoltaic systems and individuals which dwell in modelling the solar radiation on earth. It computes the location of the sun based on Bretagnon's VSOP 87 theory. It calculates the sun's azimuth with respect to a point on earth and the sun's altitude with respect to the horizon. Direct radiation is calculated and is valued in watts per square meter. It takes into account the light scattered by the atmosphere, but it uses an atmospheric model based on United States' data. PySolar has been validated against similar ephemeris codes by the United States Naval Observatory (USNO). Both azimuth and altitude estimations are within less than 0.1 degrees on average with the observations, which ensures accuracy in the estimation of solar irradiance (Stafford, 2017).

\section{METHODOLOGY}

Figure 3 shows the general methodology. The methodology is split mainly into three parts: (1) pre-processing, (2) processing, and (3) post-processing. Pre-processing prepares the necessary points needed for processing via QGIS. Processing takes the preprocessed points and runs it through python scripts, creating the building model and the solar radiation models. Post-processing visualizes the models using Blender, then perspective screenshots from Blender are overlaid with grids in QGIS for quantifying purposes, and finally, a spreadsheet software visualizes the quantified data into graphs, charts and tables.

\subsection{Data Sources}

(1) LiDAR derived Digital Terrain Model (DTM) and Digital Surface Model (DSM) from the Collective Strengthening of Community Awareness on Natural Disasters (CSCAND) agencies, Both DTM and DSM have 1-meter resolution with an original reference of WGS84, and (2) Building footprint polygons from Open Street Map (openstreetmap.org).

\subsection{Software, Plug-ins and Add-ons}

3.2.1 Quantum GIS: Quantum GIS (QGIS) is a free and open source geographic information system software (QGIS, 2018). The following QGIS Plug-ins were used: (1) Locate Points Along Lines, which create points along lines with a given offset and interval (Locate Points, 2018), and (2) Point Sampling, which samples polygon attributes and raster values from multiple layers at specified sampling points (Point Sampling Tool, 2018).

Blender: Blender is a free open source $3 \mathrm{D}$ creation suite. It supports modelling, rigging, animation, simulation, rendering, compositing and motion tracking, even video editing and game creation (About Blender, 2018). The Blender Add-on, Blender GIS was used. The add-on bridges geographic data and Blender (BlenderGIS, 2018).



Figure 3. General Methodology of the Research

\subsection{Programming Language and Modules}

Python is an object-oriented programming language that supports modules and packages. Its interpreter and extensive standard library are available without any charge and can be freely distributed (Python, 2018). The following modules where used: (1) PySolar, which simulates irradiation at any point on the surface of the earth by the sun (PySolar, 2018), and (2) Python Shapefile (PyShp), which reads and writes ESRI shapefiles in Python. The Shapefile format is a popular GIS vector data format created by ESRI (PyShp, 2018).

\subsection{Pre-Processing}

3.4.1 NDSM Generation: Using QGIS Raster Calculator, the difference between the values of the LiDAR derived Digital Surface Model (DSM) and Digital Terrain Model (DTM) results the Normalized Digital Surface Model (NDSM), which contains the heights of the features inside the extents of the raster. 


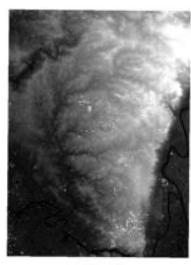

DSM
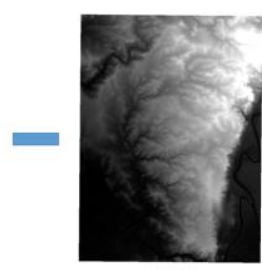

DTM

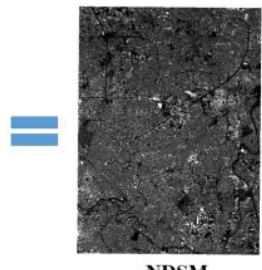

NDSM
Figure 4. NDSM Generation Process

3.4.2 Generation of Points: QGIS Regular Points vector research tool generates the ground points. The extents of the radius of the coverage of ground points is the distance of the longest point from the centroid of the buildings of interest with an additional distance of the maximum height of the tallest building to ensure that all of the buildings will be covered in the process. Points for the building are from ground points clippings by building footprint polygons and from points along the boundary using the QGIS plug-in: Locate Points Along Lines.

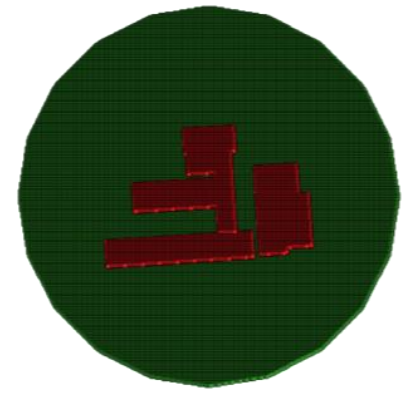

Figure 5. Ground Points (Green) and Building Points (Red) Generated in QGIS

3.4.3 Adding Attributes: Having an original projection of UTM Zone $51 \mathrm{~N}, \mathrm{X}$ and $\mathrm{Y}$ coordinates (in meters) are added to the points' attributes using the field calculator in QGIS. The points are transformed to WGS84 coordinates in order to add Longitude and Latitude values to the attribute table, then the points are transformed back to UTM Zone $51 \mathrm{~N}$.

3.4.4 Point Sampling: Elevation and height values are extracted from the LiDAR derived DTM and the generated NDSM, respectively. They were then added to the attribute table of the points using the QGIS plug-in: Point Sampling.

\subsection{Processing}

3.5.1 Building Model Generation: The pre-processed point shapefiles serve as input in a python script which creates the point shapefile for the building model. The script works in the order described in the next paragraph.

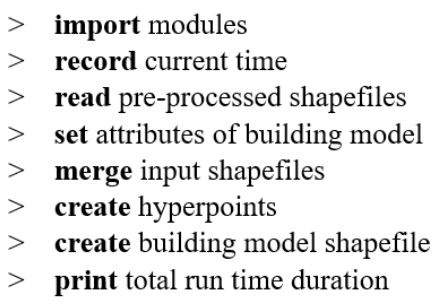

Figure 6. Pseudocode for the Python Script that Generates the Building Model
(1) The following python modules are imported: shapefile (PyShp) and datetime, these modules are called throughout the script. (2) The start time of the script runtime is recorded; this would be used latter on to calculate the total script runtime duration. (3) The shapefiles are read; these shapefiles are the points containing the building footprint boundary points and the regular points inside the extents of the building footprint polygon that was pre-processed. (4) The following attributes are then set for the output shapefile of the building model such as the $\mathrm{X}, \mathrm{Y}$, $\mathrm{Z}$, latitude, and longitude coordinates. (5) All the points from the two input shapefiles are merged, and then hyper points are created. (6) All hyper points are merged to a single list and the Z values are adjusted making sure its base value is the elevation from the DTM with 1-meter intervals extracted from the heights in the generated NDSM. (7) From the list containing all of the hyper points, values are distributed to a shapefile from the attributes laid out earlier. The shapefile is then written and saved. The output is the building model, as shown in Figure 6. (8) Having recorded the initial time of the script runtime, at the end of the script process the total duration of the script runtime is printed. The building model script runs for less than a minute.

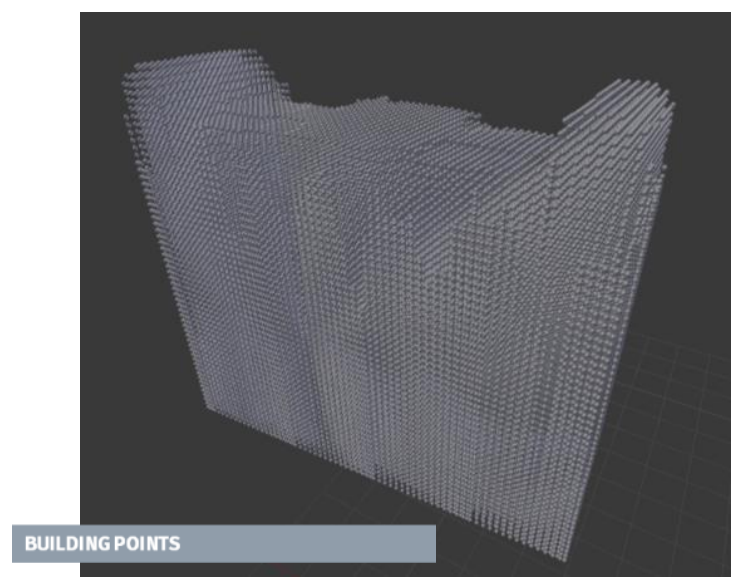

Figure 7. Generated Building Model Screenshot

3.5.2 Building Radiation Model: The output of the previous python script, the building model shapefile, and the preprocessed ground points are the input for the next python script, which records the points that gets hit by solar radiation and calculates the value of solar irradiance at a given date and time. The script works in the order described in the next paragraph.

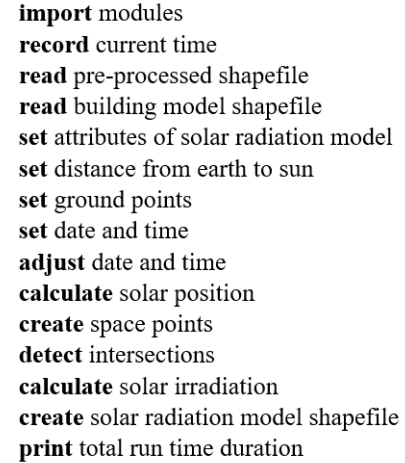

Figure 8. Pseudocode for the Python Script that Generates the Solar Irradiation Models 
(1) The following python modules are imported: shapefile (PyShp), datetime, pytz, Pysolar, and math, these modules are called throughout the script. (2) The start time of the script runtime is recorded; this would be used latter on to calculate the total script runtime duration. (3) The shapefiles are read, these are the building model, generated by the previous code, and the regular points of the area of interest that was pre-processed. (4) The following attributes are then set for the output shapefile of the building model such as the $\mathrm{X}, \mathrm{Y}, \mathrm{Z}$, latitude, and longitude coordinates, and solar irradiance values. (5) The distance from the Earth to the sun is set at approximately $150,000,000,000$ meters. (McMordie, 2012) (6) Ground points are set using the regular points of the area of interest generated in the preprocessing of the data. (7) The date and time are set, these are needed in computing for solar altitude, azimuth, and irradiance. The inputs required consists of the year, month, day, hour, minute, and second. The date and time of the research ranges from January to December, every first day of the month, and from 08:00 to 17:00 (which is described as the normal working hours of government bodies in the Philippines according to Executive Order No. 292). This is manually changed every time the code is run, for a different date and time input. (8) The time zone is then adjusted to Coordinated Universal Time (UTC) from Philippine local time using built-in modules. Since Pysolar reads the input time in UTC format. (9) Using Pysolar and the adjusted date and time as input, the solar azimuth and solar altitude is calculated. Using the solar angles and the distance from earth to sun to compute the differences between a point from the ground to an equally translated point counterpart in space.

$$
\begin{aligned}
& \mathrm{x}=\mathrm{x}_{0}+\mathrm{at} \\
& \mathrm{y}=\mathrm{y}_{0}+\mathrm{bt} \\
& \mathrm{z}=\mathrm{z}_{0}+\mathrm{ct}
\end{aligned}
$$

where

$$
\begin{aligned}
& (x, y, z)=\text { any point on the line } \\
& \left(x_{0}, y_{0}, z_{0}\right)=\text { known point on the line } \\
& t<a, b, c\rangle=\text { some vector along the the line }
\end{aligned}
$$

(10) Applying all the computed differences to all ground points, the equally translated counterpart space points are created. The building model generated from the previous code is loaded. Using a set of parametric equations of a line in 3D space as shown in Equation 1 in the code, all the intersections on the building model of the line from the ground point and its counterpart space point is collected. (11) From the pool of intersected points, points that have the shortest distance from their respective space points of the line of intersection is recorded, this indicates that it is the first point a solar ray touches and that light will not pass through, also factoring in shadowing effects. (12) Direct clear-sky solar irradiation is then computed using PySolar. Then, irradiation is appended to each intersected point. (13) From the list containing the solar radiation points and the list containing solar irradiance, values are distributed to a shapefile from the attributes laid out earlier. The shapefile is then written and saved. The outputs are the radiation models of the building at a given date and time. (14) Having recorded the initial time of the script runtime, at the end of the script process the total duration of the script runtime is printed. If ran individually the script runs for 3-5 hours, and If ran simultaneously (in this research the laptop can support 12 codes running at the same time) the code runs for 8-10 hours.

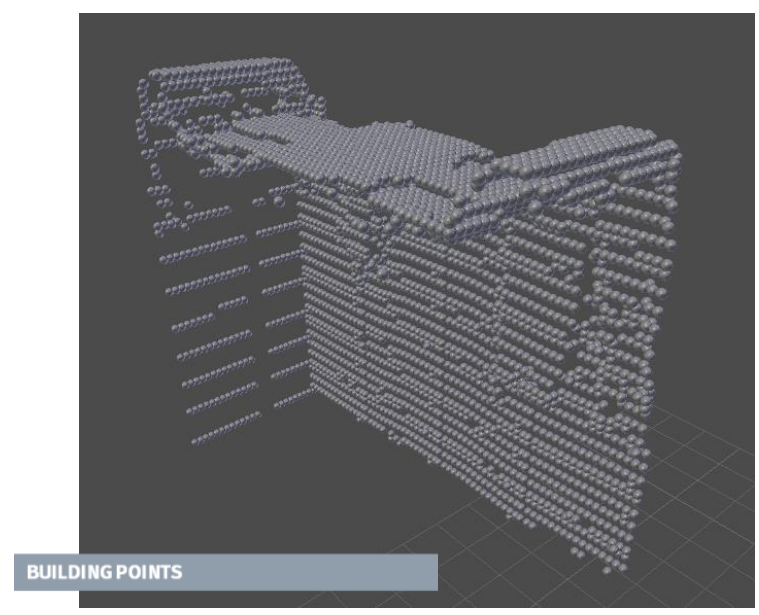

Figure 9. Generated Solar Irradiation Model Screenshot

\subsection{Post-Processing}

3.6.1 Model Visualization: The building model shapefile and the solar radiation models are loaded and visualized in blender. Screenshots are taken at specific positions, mainly the front, back, left, right and roof (top) faces of the building.

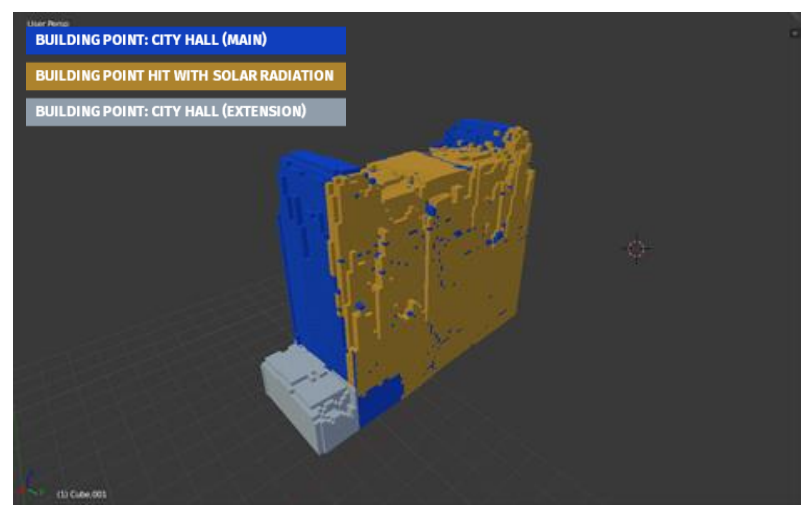

Figure 10. Building Model and Solar Irradiation Model Screenshots

3.6.2 Conversion to Grid: The screenshots are then converted into a gridded format in QGIS for quantifying purposes. This is done by overlaying a grid (with a grid factor of 25) over the screenshot and manually checking if there are any parts of the solar irradiation model in a box of the grid.

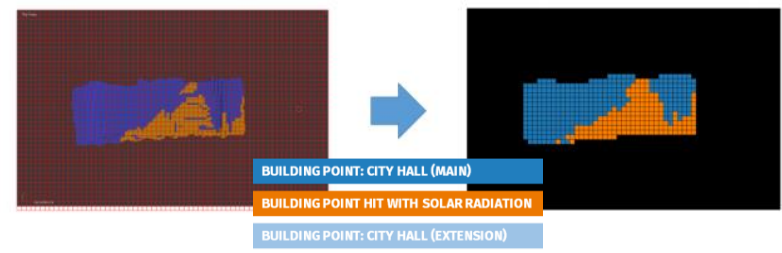

Figure 11. Conversion of the Screenshot of the Model to a Gridded Version 
3.6.3 Creation of Tables and Graphs: Numerical values computed from the codes and extracted from the gridded version of the screenshots are recorded in a spreadsheet program. The spreadsheet program is used to analyse the data and to produce graphs, charts, and tables, that will help visualize the numerical data.

\section{RESULTS AND DISCUSSION}

\subsection{Solar Irradiance}

For the year 2017 average solar irradiation values ranges from $800 \mathrm{~W} / \mathrm{m}^{2}$ to $1000 \mathrm{~W} / \mathrm{m}^{2}$. However, at 17:00 average solar irradiation values drop to $500 \mathrm{~W} / \mathrm{m}^{2}$. Solar irradiation values have little deviation during 8:00 to 16:00 showing that it almost has a constant effect in computing solar irradiation values in the surface of the building through-out those hours.

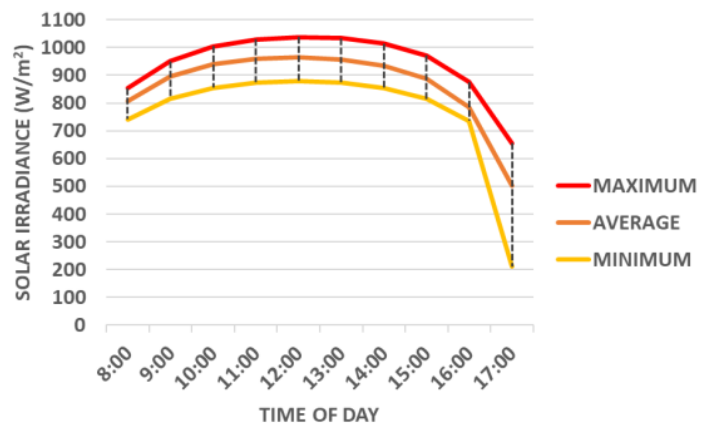

Figure 12. Hourly Solar Irradiation (2017)

Solar irradiation reaches its maximum at February and its minimum at July. Although there is not much deviation in the average solar irradiation values all through-out the year. Which shows that average solar irradiation values also have an almost constant effect in computing solar irradiation values in the surface of the building through-out the year.

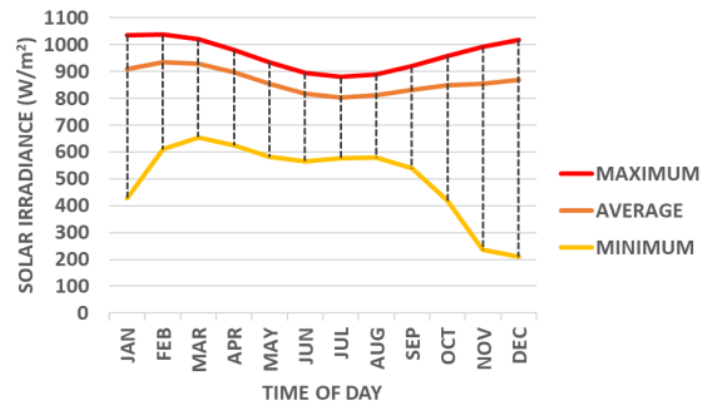

Figure 13. Monthly Solar Irradiation (2017)

Since solar irradiance values do not change drastically throughout the chosen period of time, whether monthly or hourly, defining the covered surface area that is hit by solar radiation is sufficient to define the photovoltaic potential of a face of a building, or the building itself.

\subsection{Surface Receiving Radiation}

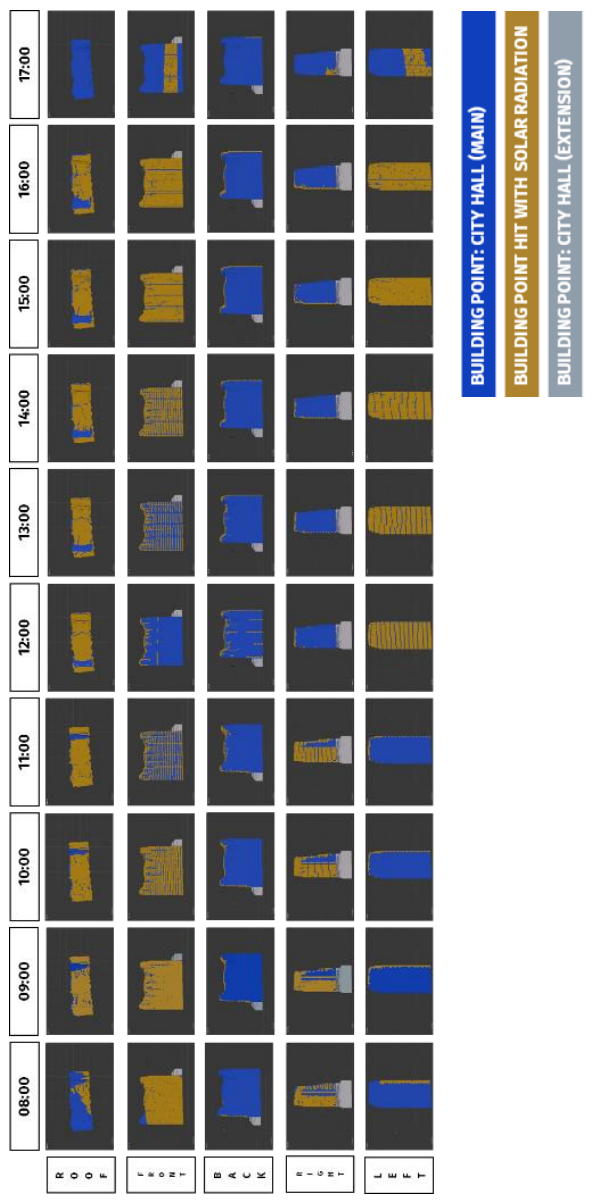

Figure 14. Screenshots of the Solar Radiation Model Visualized in Blender (January 01, 2017)

From 8:00 to 16:00 all faces of the city hall receive solar radiation, at 17:00 some faces of the building do not receive solar radiation. These can be seen in Figure 14, which is a sample of a set of model screenshots for January 01, 2017. This shows that all faces of the building are possible contributors for a good photovoltaic potential of Quezon City Hall.
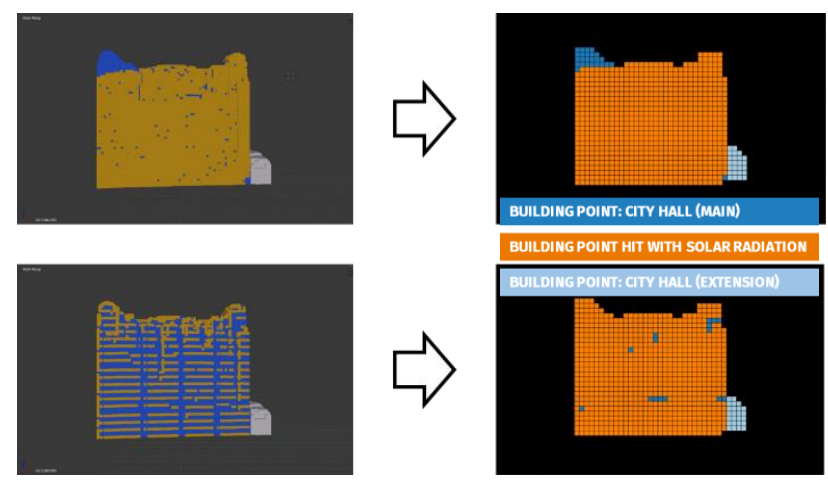

Figure 15. Screenshot of Model to Gridded Image

A converted version of Figure 14 to a gridded format is shown in Figure 16 for comparison. 


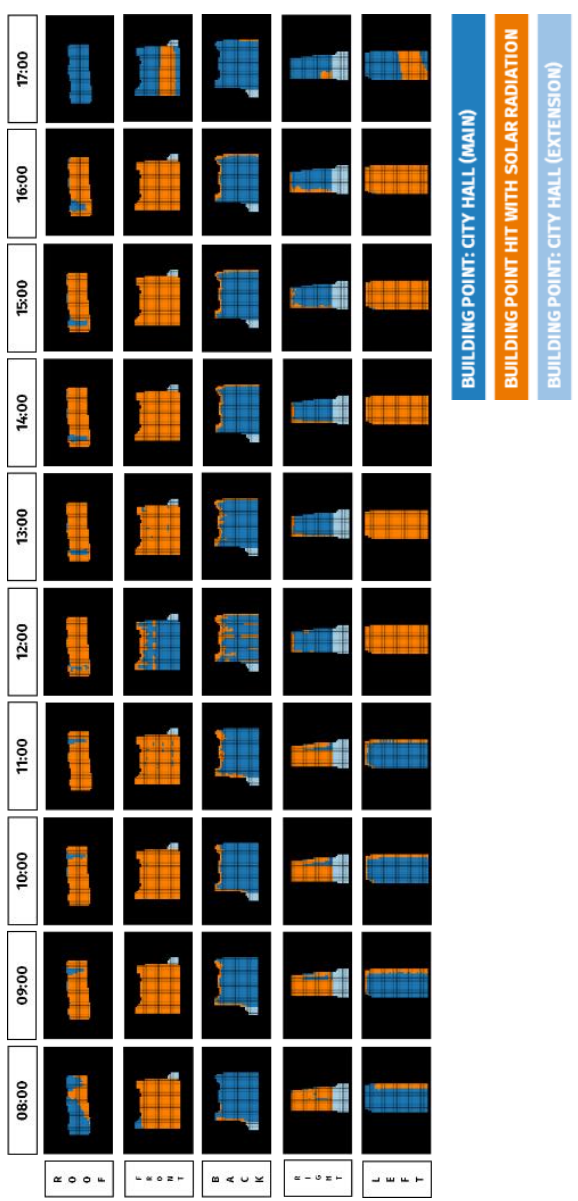

Figure 16. Screenshots of Gridded Solar Radiation Model Visualized in Blender (January 01, 2017)

From 8:00 to 16:00 the front face of the city hall has the largest surface area that receives solar radiation. At 12:00, on average, the roof is the face of the building that receives the most solar radiation. Which means the front face of the building contributes the most solar irradiance than the rest of the faces of the building.



Figure 17. Hourly - Surface Receiving Radiation (2017)

From 8:00 to 16:00 all through-out the year average values of solar irradiation on the roof almost remain at a constant value, this can be brought on by the limitation of the roofs area. It can be shown from Figure 17 that all through-out the day the roof almost fully receives solar radiation. For the back and the roof, solar irradiation values peaks at the middle of 8:00 to 12:00 and 12:00 to $16: 00$ and reaches its minimum at 12:00. For the right and left faces of the building, solar irradiation value trends seem to be inversely proportional with respect to each other, as when values in the right face dwindle the value in the left face rise. This shows the probable positioning trend of the sun with respect to the Quezon City Hall and can be used for rough estimates of surfaces which receive solar radiation for nearby buildings.

From May to August the surface of the city hall which receives solar radiation is the back, and the front for the rest of the months. All throughout the year the roof receives less solar radiation than the front and the back faces of the building, but the roof receives more solar radiation than that of the left and right faces. Which shows that the walls contribute more than the roof in terms of the building's photovoltaic potential.

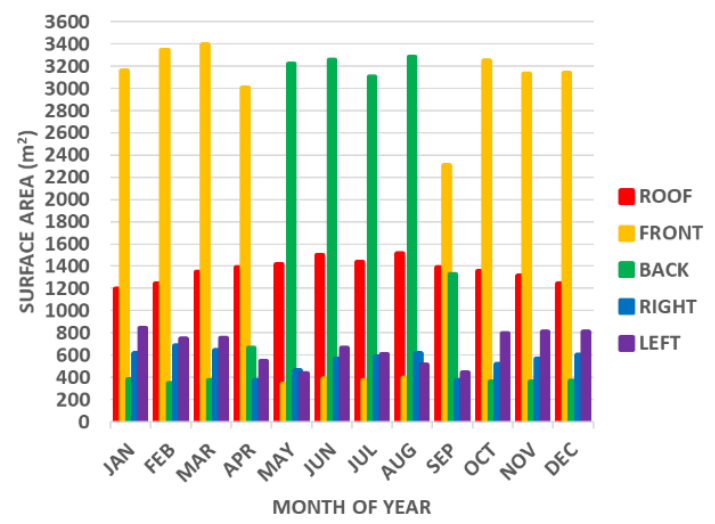

Figure 18. Monthly - Surface Receiving Radiation (2017)

It shows that solar irradiation value trends for the roof, left and right faces of the building are almost constant while trends for the front and back faces show large deviations in certain times of the day. With additional yearly observations this can be used to predict the position of the sun relative to the city hall.

In total, the ratio of roof to walls for the city hall in receiving solar radiation is $22 \%$ to $78 \%$ respectively. Which shows that solar irradiance in the walls is $3.5 \mathrm{x}$ that of the roof, and shows the importance of the walls in contributing to the city hall's photovoltaic potential.

\section{CONCLUSION AND RECOMMENDATIONS}

The research has shown that Quezon City Hall has good photovoltaic potential since: (1) the amount of solar irradiation received in 2017 ranges from $800 \mathrm{~W} / \mathrm{m}^{2}$ to $1000 \mathrm{~W} / \mathrm{m}^{2}$ on average from 08:00 to 16:00, (2) all faces of the building receive solar radiation in a day, and (3) the wall's surface area that receive solar radiation is $3.5 \mathrm{x}$ more than what the roof receives. This means that all sides of the building can be proper candidates for installation of solar panels or Building Integrated Photovoltaics (BIPV). For further improvement, the research recommends: (1) re-running the python script on a more detailed building model of Quezon City Hall, (2) including vegetation and other infrastructures as shadowing factors, (3) considering diffused radiation by including cloud cover and atmospheric conditions, (4) creating a more precise methodology to quantify the coverage area where radiation hits, (5) improving the code that generates solar radiation models so it will run at less than 35 hours for individual runs and less than 8-10 hours for 12 simultaneous runs, and (6) revisiting the whole methodology since the development of QGIS 3.0, which has more 3D capabilities, is ongoing and is still open source. 


\section{REFERENCES}

Adeleke, A. K., \& Smit, J. L., 2016: Integration of LiDAR Data with Aerial Imagery for Estimating Rooftop Solar Photovoltaic Potentials in City of Cape Town. The International Archives of the Photogrammetry, Remote Sensing and Spatial Information Sciences, XLI-B7, 617-214.

Agugiaro, G., Remondino, F., Stevanato, G., De Filippi, R., \& Furlanello, C., 2011: Estimation of Solar Radiation on Building Roofs in Mountainous Areas. The International Archives of the Photogrammetry, Remote Sensing and Spatial Information Sciences, 38, 155-160.

Beseničar, J., Trstenjak, B., \& Setnika, D., 2008: Application of Geomatics in Photovoltaics. The International Archives of the Photogrammetry, Remote Sensing and Spatial Information Sciences, XXXVII Part B4, 53-56.

Blender Development Team, 2018. Blender Version 2.7. https://www.blender.org/about. (2018)

Blender GIS Development Team, 2018. Blender GIS. https://github.com/domlysz/BlenderGIS. (2018)

Brito, M., Guimaraes, S., Catita, C., \& Redweik, P., 2017: The Importance of Facades for the Solar PV Potential of a Mediterranean City using LiDAR Data. ELSEVIER: Renewable Energy, 85-94.

Chwieduk, D., 2014: Solar Energy in Buildings - Thermal Balance for Efficient Heating and Cooling. Elsevier Inc.

Debek, L., 2018. Locate points along lines 0.3. https://plugins.qgis.org/plugins/LocatePoints/. (2018)

International Renewable Energy Agency, 2017: Renewables Readiness Assessment: The Philippines. International Renewable Energy Agency.

Jaugsch, F., \& Lowner, M. O., 2016: Estimation of Solar Energy on Vertical \#D Building Walls on City Quarter Scale. The International Archives of the Photogrammetry, Remote Sensing and Spatial Information Sciences, XLII-2/W2, 135-143.

Jurgiel, B., 2018. Point sampling tool 0.4.2. https://plugins.qgis.org/plugins/pointsamplingtool/. (2018)

Kanters, J., Wall, M., \& Kjellsson, E., 2014: The Solar Map as a Knowledge Base for Solar Energy Use. International Conference on Solar Heating and Cooling for Buildings and Industry, 15971606.

Martin, A. M., Dominguez, J., \& Amador, J., 2015: Applying LiDAR Datasets and GIS Based Model to Evaluate Solar Potential over Roofs: A Review. AIMS: Energy, Vol. 3 Issue 3, 326-343.

McMordie, R. K., 2012: SOLAR ENERGY FUNDAMENTALS. The Fairmont Press, Inc.

Miglani, S., Orehounig, K., \& Carmaliet, J., 2015: A Method for Generating Hourly Solar Radiation on Building Rooftops Accounting for Cloud Cover Variability. CISBAT, 717-722.
Patil, N. B., \& Nalawade, S. S., 2016: Applications of GIS to Smart Cities. International Journal of Recent Advances in Engineering \& Technology, 2347-2812.

PyShp Development Team, 2017. PyShp 1.2.12 https://github.com/GeospatialPython/pyshp. (2018)

Python Development Team. (2018). Python 2.7. https://www.python.org/doc/essays/blurb/Python. (2018)

QGIS Development Team, 2017. QGIS 2.18 Las Palmas. https://qgis.org/en/site. (2018)

Quezon City Government, 2018. Facts and Figures. https://quezoncity.gov.ph/index.php/facts-and-figures. (2018)

Quezon City Government, 2018. Locus of Govevrnment Operations. https://quezoncity.gov.ph/index.php/locus-ofgovernment-operations. (2018)

Rafiee, A., Dias, E., Fruijtier, S., \& Scholten, H., 2014: From BIM to Geo-analysis: View Coverage and Shadow Analysis by BIM/GIS Integration. 12th International Conference on Design and Decision Support Systems in Architecture and Urban Planning, 397-402.

Stafford, B., 2017. PySolar 0.6. http://pysolar.org/. (2018)

United Nations, 2016: The Sustainable Development Agenda. http://www.un.org/sustainabledevelopment/developmentagenda. (2018)

Wegertseder, P., Lund, P., Mikkola, J., \& Alvarado, R., 2016: Combining Solar Resource Mapping and Energy System Integration Methods for Realistic Valuation of Urban Solar Energy Potential. ELSEVIER: Solar Energy, 325-336.

Wieland, M., Nichersu, A., Murshed S, \& Wendel, J., 2015: Computing Solar Radiation on CityGML Building Data. AGILE. 